\title{
Does prehabilitation modify muscle mass in patients with rectal cancer undergoing neoadjuvant therapy? A subanalysis from the REx randomised controlled trial
}

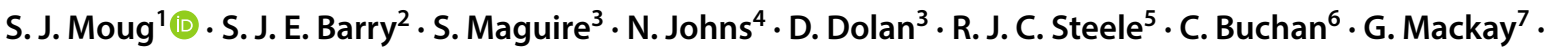 \\ A. S. Anderson ${ }^{8} \cdot$ N. Mutrie ${ }^{9}$
}

Received: 28 April 2020 / Accepted: 10 June 2020 / Published online: 20 June 2020

(c) The Author(s) 2020

\begin{abstract}
Background Patients with rectal cancer who present with sarcopenia (low muscle mass) are at significantly greater risk of postoperative complications and reduction in disease-free survival. We performed a subanalysis of a randomised controlled study [the REx trial; www.isrctn.com; 62859294] to assess the potential of prehabilitation to modify muscle mass in patients having neoadjuvant chemoradiotherapy (NACRT).

Methods Patients scheduled for NACRT, then potentially curative surgery (August 2014-March 2016) had baseline physical assessment and psoas muscle mass measurement (total psoas index using computed tomography-based measurements). Participants were randomised to either the intervention (13-17-week telephone-guided graduated walking programme) or control group (standard care). Follow-up testing was performed 1-2 weeks before surgery.

Results The 44 patients had a mean age of 66.8 years (SD 9.6) and were male (64\%); white (98\%); American Society of Anesthesiologists class 2 (66\%); co-morbid (58\%); overweight (72\%) (body mass index $\geq 25 \mathrm{~kg} / \mathrm{m}^{2}$ ). At baseline, $14 \%$ were sarcopenic. At follow-up, 13 (65\%) of patients in the prehabilitation group had increased muscle mass versus 7 (35\%) that experienced a decrease. Conversely, $16(67 \%)$ controls experienced a decrease in muscle mass and $8(33 \%)$ showed an increase. An adjusted linear regression model estimated a mean treatment difference in Total Psoas Index of $40.2 \mathrm{~mm}^{2} / \mathrm{m}^{2}$ (95\% CI -3.4 to 83.7$)$ between groups in change from baseline $(p=0.07)$.

Conclusions Prehabilitation improved muscle mass in patients with rectal cancer who had NACRT. These results need to be explored in a larger trial to determine if the poorer short- and long-term patient outcomes associated with low muscle mass can be minimised by prehabilitation.
\end{abstract}

Keywords Prehabilitation $\cdot$ Muscle wasting $\cdot$ Rectal cancer $\cdot$ Sarcopenia $\cdot$ Neoadjuvant chemoradiotherapy $\cdot$ Sarcopenia . Neoadjuvant therapy $\cdot$ Rectal neoplasms $\cdot$ Preoperative care $\cdot$ Walking

S. J. Moug

susanmoug@nhs.net

1 Department of Surgery, Royal Alexandra Hospital, Corsebar Road, Paisley PA2 9PN, UK

2 Department of Mathematics and Statistics, Strathclyde University, Glasgow, UK

3 University of Glasgow, Glasgow, UK

4 Western General Hospital, Crewe Road South, Edinburgh, UK
Ninewells Hospital and Medical School, Dundee, UK

6 Department of Surgery, Royal Alexandra Hospital, Paisley, UK

7 Department of Surgery, Glasgow Royal Infirmary, Glasgow, UK

8 Division of Cancer Research, Ninewells Medical School, Dundee, UK

9 Institute of Sport, Physical Education and Health Sciences, Moray House School of Education, Edinburgh, UK 


\section{Introduction}

Low muscle mass, or sarcopenia, has been reported in up to $60 \%$ of patients with colorectal cancer either at the time of diagnosis, or as a result of neoadjuvant chemoradiotherapy (NACRT) [1-4]. If present, the patient is at higher risk of worse outcomes following surgical resection, including major complications, increased hospital stay and early postoperative mortality [5, 6]. Sarcopenia also appears to influence a patient's long-term outcome with reduced 1- and 5-year survival in patients with colorectal cancer reported [7, 8]. These poorer outcomes are not restricted to patients with rectal cancer and have been extensively reported in other malignancies including, renal, lung and gastroesophageal [9-12]. However, to date, there are no reported interventions that have modified or offset treatment-related sarcopenia in these cancer populations.

Prehabilitation is an intervention that places emphasis on optimising patients prior to their first treatment and consists predominately of individualised physical activity or exercise programmes. Shown to be safe and feasible in many cancer populations, prehabilitation studies are now reporting on improved patient outcomes after surgery [13-22]. With physical activity and exercise providing physiological overload that increases muscle mass in the general population, prehabilitation in patients with rectal cancer provides an opportunity to determine if low muscle mass can be modified.

Within a study that aimed to assess the feasibility of performing a physical activity intervention during NACRT for rectal cancer, we performed a subanalysis to establish the prevalence of sarcopenia and to determine if a physical activity intervention had the potential to modify muscle mass.

\section{Materials and methods}

\section{Study design and data collection}

From August 2014 to March 2016 (20 months), any adult over 18 years with a new diagnosis of rectal cancer where NACRT was planned was considered for inclusion in the REx trial [23]. The trial was approved by the West of Scotland Research Ethics Service (14/WS/0079) and registered with ISRCTN (www.isrctn.com; 62859294; 17th March 2014). This study was funded by the Chief Scientist Office (CZH/4/986; www.cso.scot.nhs.uk).

This was a two-arm randomised controlled feasibility study (RCT) with the full protocol, physical intervention and results published [23]. Briefly, each participant's demographics were recorded and they completed baselinetesting including daily step count, physical parameters and psychological. Clinico-pathological outcomes were also recorded. Baseline testing was pre-intervention (Test 1 prior to undergoing NACRT) and repeated post-intervention (1-2 weeks pre-surgery, post-prehabilitation, Test 2).

The walking programme started prior to NACRT and was of minimum 13-week duration. It consisted of graduated step count goals using pedometers and was telephone guided supplemented by walking diaries. The target was for the participants to increase their average daily step count by 3000 accumulated above their baseline by week 8 [24-28]. The control group received standard care and told to maintain their normal level of physical activity.

\section{Body composition measurement}

Muscle mass was measured on each patient using a validated technique: the total cross-sectional area of the psoas muscles [total psoas area, (TPA)] [8]. This was measured manually using a free-hand drawing technique on Picture Archiving and Communication System (PACS) at the level of the L3 vertebra on pre-treatment computed tomography (CT) scan. To ensure standardisation, the exact level of measurement was defined as the CT slice in which both transverse processes were maximally in view. The outline of each individual psoas muscle was traced, the area of each calculated, and summated to provide the TPA $\left(\mathrm{mm}^{2}\right)$. The TPA was then standardised for patient height using the formula TPA $\left(\mathrm{mm}^{2}\right) /$ height $\left(\mathrm{m}^{2}\right)$ to provide the total psoas index (TPI) for each patient. TPI was calculated pre-NACRT and postNACRT within 4 weeks of planned surgery.

The threshold values used for the diagnosis of sarcopenia were: TPI less than $524 \mathrm{~mm}^{2} / \mathrm{m}^{2}$ for males, and $385 \mathrm{~mm}^{2} /$ $\mathrm{m}^{2}$ for females [29]. To ensure the reliability of our technique, 20 scans were randomly selected and measured for sarcopenia by blinded trained investigators to allow calculation of inter- and intra-class correlation coefficients (ICC). The ICC $\left(r^{2}\right)$ values for inter- and intra-class reliability were 0.957 and 0.985 , respectively (close to 1 indicates excellent agreement).

\section{Statistical analysis}

The Mann-Whitney test was used to test for a difference in median change from baseline in TPI between study groups, while the chi-squared test was used to compare the equivalent categorical variable defining sarcopenia (level of significance $p<0.050$ ). A linear regression model was fitted to the change from baseline in TPI to assess its association with the study group while adjusting for baseline variables. Spearman's correlation coefficient was calculated to assess the association between change in TPI and step count. No 
hypothesis tests were carried out for this part of the analysis due to small numbers. All statistical analysis were undertaken using R (version 3.5.0) [30].

\section{Results}

\section{Baseline participant characteristics}

A total of 48 patients ( $n=24$ in each group) were recruited to the study, of which 44 underwent follow-up CT staging (the 4 who did not were all in the intervention group). The 44 patients had a mean age of 66.8 years (SD 9.6). The majority were male (64\%), white (98\%), American Society of Anesthesiologists (ASA) class $2(66 \%)$ and co-morbid (58\%). Most (59\%) participants currently or had previously smoked, $86 \%$ reported current alcohol consumption and $72 \%$ were overweight (body mass index (BMI) $\geq 25 \mathrm{~kg} / \mathrm{m}^{2}$ ) with $20 \%$ obese $\left(\mathrm{BMI} \geq 30 \mathrm{~kg} / \mathrm{m}^{2}\right)$.

Overall, $6(n=14 \%)$ of the 44 patients were sarcopenic at baseline: intervention group 4 (20\%) versus control group $2(8 \%)$. Median baseline TPI in the intervention group was $583.5 \mathrm{~mm}^{2} / \mathrm{m}^{2}$, and in the control $593.3 \mathrm{~mm}^{2} / \mathrm{m}^{2}$ (Table 1 ).

None of the 44 participants achieved the recommended government activity guidelines at baseline. The mean number of steps per day of all participants was 6248 (range $1151-17,422$ steps) leading to $54 \%$ classified as sedentary or only slightly active (Table 1 ).

\section{Follow-up results: step count}

Median walking intervention duration was 14 weeks (IQR 13-17). At follow-up testing, both groups recorded a reduction in daily step count, with the control group showing a larger reduction (difference between groups in change from baseline of 785 [95\% CI - 1194, 2765] adjusted for baseline median daily step count, age and sex). A higher percentage of the intervention group achieved step count improvements at 12 weeks (23.5\% versus $15.8 \%$ ) [19].

\section{Follow-up results: muscle mass measurement}

The intervention group showed a median increase in TPI of $16.0 \mathrm{~mm}^{2} / \mathrm{m}^{2}$ to a median TPI of $624.2 \mathrm{~mm}^{2} / \mathrm{m}^{2}$, compared to a decrease of $8.4 \mathrm{~mm}^{2} / \mathrm{m}^{2}$ in the control group to a median TPI of $571.2 \mathrm{~mm}^{2} / \mathrm{m}^{2}$ (group difference in median change from baseline of $24.4 \mathrm{~mm}^{2} / \mathrm{m}^{2}, p=0.07$ ) (Fig. 1). Figure 2 displays the scatter plots for TPI showing that $13(65 \%)$ of patients in the prehabilitation group experienced an increase in muscle mass, versus 7 (35\%) that experienced an overall decrease, while 16 (67\%) controls experienced a decrease and $8(33 \%)$ showed an increase $(p=0.07)$.

A linear regression model adjusting for age, any comorbidities and baseline TPI estimated a mean treatment difference of $40.2 \mathrm{~mm}^{2} / \mathrm{m}^{2}$ (95\% CI - 3.4 to 83.7 ) between the intervention and control groups in change from baseline $(p=0.07)$.

Table 1 Comparison of baseline physical measurements of REx trial participants: Intervention group versus control group, for patients with muscle mass measurements

\begin{tabular}{lccc}
\hline & All $(N=44)$ & Intervention $(N=20)$ & Control $(N=24)$ \\
\hline Mean TPI $\mathrm{mm}^{2} / \mathrm{m}^{2}(\mathrm{SD})$ & $598(161)$ & $585(152)$ & $608(171)$ \\
Median TPI $\mathrm{mm}^{2} / \mathrm{m}^{2}(\mathrm{IQR})$ & $584(460-707)$ & $584(450-650)$ & $593(460-718)$ \\
Sarcopenic & & & \\
$\quad$ No & $38(86 \%)$ & $16(80 \%)$ & $22(92 \%)$ \\
Yes & $6(14 \%)$ & $4(20 \%)$ & $2(8 \%)$ \\
Median steps per daya $(\mathrm{IQR})$ & $6.2(5.1-10.1)$ & $6.1(4.7-9.2)$ & $6.7(5.7-10.3)$ \\
Sedentary & $10(23 \%)$ & $6(30 \%)$ & $4(17 \%)$ \\
Slightly active & $13(30 \%)$ & $5(25 \%)$ & $8(35 \%)$ \\
Moderately active & $16(37 \%)$ & $7(35 \%)$ & $9(39 \%)$ \\
Very active & $4(9 \%)$ & $2(10 \%)$ & $2(9 \%)$ \\
Sit-to-stand test, no. completed in 30 s [mean (SD)] & $11.5(2.7)$ & $11.6(2.4)$ & $11.4(3.0)$ \\
6-Min walking tests, metres [mean (SD)] & $441.3(63.9)$ & $446.9(61.9)$ & $436.7(66.4)$ \\
\% of week spent active [mean (SD)] & $6.6(2.8)$ & $6.5(2.9)$ & $6.7(2.9)$ \\
\% of week spent sedentary [mean (SD)] & $75.9(12.1)$ & $76.9(6.1)$ & $74.9(15.6)$ \\
\hline
\end{tabular}

TPI Total Psoas Index

${ }^{\text {a } E x p r e s s e d ~ a s ~ t h o u s a n d s ~}$ 


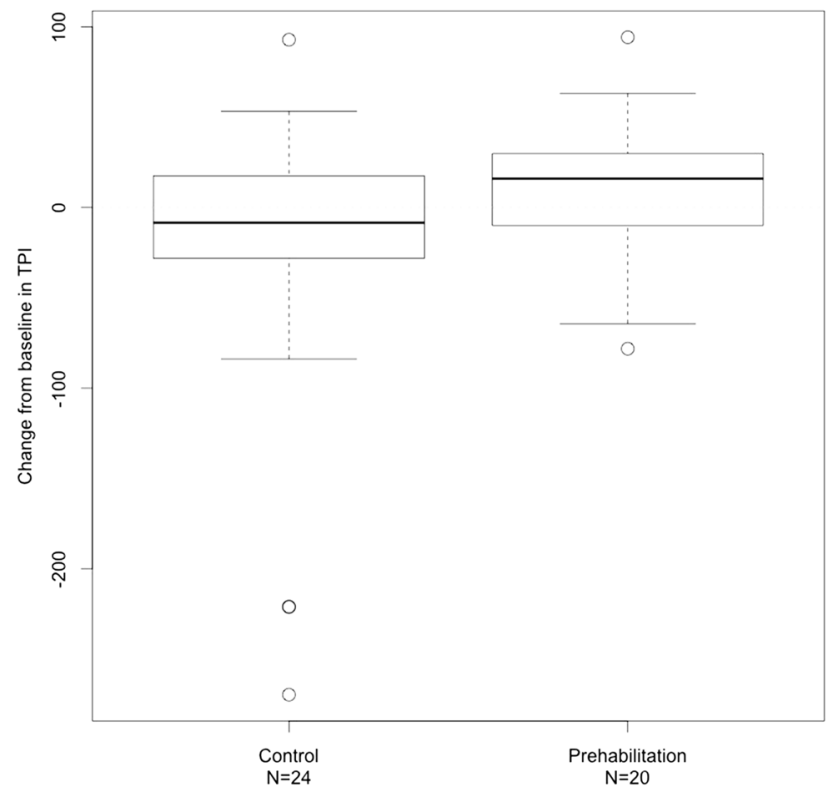

Fig. 1 Comparison of change in psoas muscle mass $\left(\mathrm{mm}^{2} / \mathrm{m}^{2}\right)$ after physical intervention in patients with rectal cancer undergoing chemoradiotherapy. TPI $=$ Total Psoas Index
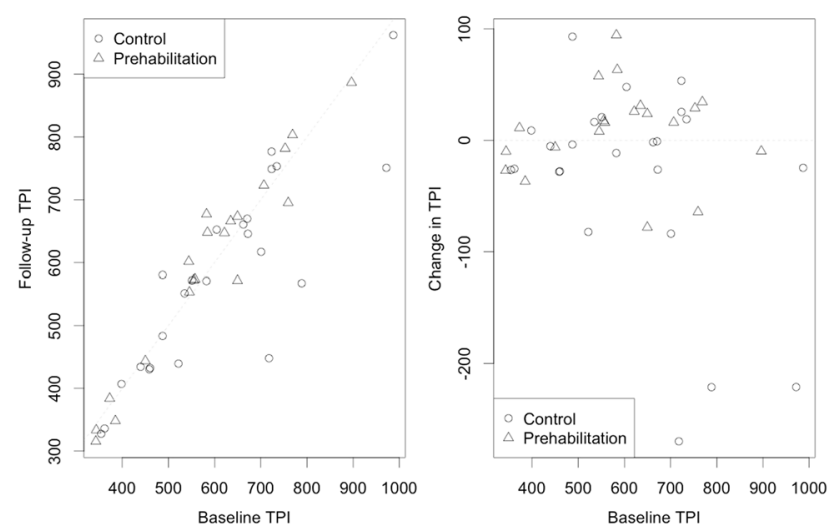

Fig. 2 Scatterplots showing Total Psoas Index (TPI) measurements $\left(\mathrm{mm}^{2} / \mathrm{m}^{2}\right)$ before and after the study period plus change from baseline in patients undergoing neoadjuvant chemoradiotherapy for rectal cancer. The dotted lines represent zero change

\section{Discussion}

This is the first study to report modification of muscle mass with prehabilitation in patients with colorectal cancer who had neoadjuvant therapy. The graduated individualised walking programme provided sufficient muscle overload to increase psoas muscle mass in $65 \%$ of the intervention group in comparison to the controls where $67 \%$ had the expected reduction in muscle mass as a consequence of having long-course chemoradiotherapy. With
$14 \%$ of patients presenting with sarcopenia at diagnosis, prehabilitation may have a further role to play in the perioperative pathway.

Body composition has increasingly been recognised as having an influencing prognostic role in colorectal cancer [5-8, 31]. Despite being modifiable, in contrast to pathological staging, there are few reported interventions to modify muscle mass and potentially improve patient outcomes in the surgical setting [32]. Furthermore, it is not routine practice to screen for low muscle mass and the results from this study show that other physical measurements (daily step count, 6MWT, STS) are not directly related to muscle mass. In particular, BMI is limited as the typical physical appearance of a patient with newly diagnosed colorectal cancer is changing with cancer cachexia becoming increasingly infrequent. This is likely to reflect both the introduction of the NHS Bowel Screening Programme detecting cancers at an earlier stage and of societal behavioural changes in diet and physical activity [33]

Evidence for screening and successful physical activity interventions comes from the gerontology literature where sarcopenia or low muscle mass has been associated with ageing. Prevention or treatment of this muscle mass reduction has been seen as key to enabling older adults to maintain their quality of life and minimise physical limitations [34]. Two recent reviews assessed various forms of exercise in older adults and concluded that resistance training had a lead role in optimising muscle mass and walking capacity $[35,36]$.

There are several proposed mechanisms for the relationship between low muscle mass and colorectal cancer outcomes. First, it could be that exercise simply provides sufficient physiological overload to improve muscle mass and, therefore, improve mobility as the patient goes through their treatment, minimising cardiorespiratory complications. Step count per se does not appear to relate to psoas muscle mass perhaps because it does not measure intensity and alternative physical capacity measurements could be considered to explore this relationship such as dynamometry. Second, systemic inflammation as a host response to the underlying rectal malignancy has been shown to result in low muscle mass [37]. Third, observational data have consistently shown that patients who are more physically active improve their cancer-specific survival leading to the hypothesis that exercise could have an anti-tumour strategy requiring a precision oncology approach $[33,38]$. Finally, the gut microbiome could be a link; alterations in its composition could reduce chronic inflammation and anabolic resistance, leading to an increased muscle mass [39].

Prehabilitation prior to surgery provides an excellent opportunity to modify muscle mass. With an increasing number of publications supporting its feasibility in the colorectal cancer setting, accompanied by increases in aerobic 
capacity and potentially a reduction in complications, the perioperative care pathway is changing [13-22]. The components of prehabilitation vary between studies with an initial focus on increasing aerobic capacity now expanding to consider psychological and dietary interventions alongside resistance training as stated in the recent Macmillan Prehabilitation Evidence and Insight Review [40]. There is still much work to be done in identifying the optimal prehabilitation pathway for each individual patient, including what components constitute their prehabilitation, what duration and intensity and in what setting: hospital, community and self-led.

There are strengths to this study. We prospectively recruited a population that is often under-reported in research studies, but one that reflects many current surgical practices: older, co-morbid, predominately sedentary, deprived and overweight. With $83 \%$ of these participants completing the prehabilitation study, this population should not be excluded from future work. In addition, muscle mass measurement was shown to be a valid, simple and reproducible technique that could be integrated into the NHS with minimal costs.

The authors acknowledge limitations. This was an unplanned subanalysis of a feasibility study so the reader must be cautious about making inferences about the reported outcomes due to very small numbers. In addition, selection bias cannot be excluded with motivated patients potentially more likely to participate and, therefore, achieve better results.

\section{Conclusions}

Patients with rectal cancer presenting with low muscle mass and those developing it as a result of NACRT have poorer short- and long-term patient outcomes as a consequence. This study tentatively reports that a targeted individualised physical activity intervention (prehabilitation) may increase muscle mass and offset the expected reduction with NACRT. This novel work needs to be explored in a larger trial setting to determine the influence of prehabilitation on short- and long-term patient outcomes.

Acknowledgements Research Nurses at the Clinical Research Facility Glasgow (including Laura Rooney and Steven Henderson), Dr Carly Bisset (West of Scotland Core Surgical Trainee); the Colorectal Cancer Nurse Specialists of Greater Glasgow and Clyde; the department of physiotherapy, Royal Alexandra Hospital, Paisley, and all the participants of this study who kindly donated their time and enthusiasm. We would also like to thank Professor Ken Fearon for his support for this work.

Author contributions SJM, ASA, NM, RJCS, SJEB, GM and CBu led the development and design of the study. SJM and SM led the data collection. SM, NJ, DD and SJEB analysed the data. SJM, SJEB,
SM, ASA and NM interpreted the data. SJM, SJEB, NM, GM, RJCS, $\mathrm{DD}, \mathrm{CBu}$ and ASA led the drafting and final submission. All authors approved the final manuscript.

Funding This study was funded and supported by the Chief Scientist Office (CZH/4/986) www.cso.scot.nhs.uk

\section{Compliance with ethical standards}

Conflict of interest The authors declare no conflicts of interest. Interim findings were presented as an oral presentation at the Association of Coloproctology of Great Britain and Ireland, Birmingham, UK, July 2018.

Ethical approval The trial was approved by the West of Scotland Research Ethics Service (14/WS/0079) and registered with ISRCTN (www.isrctn.com, www.cso.scot.nhs.uk).

Informed consent Informed consent was obtained from all individual participants.

Open Access This article is licensed under a Creative Commons Attribution 4.0 International License, which permits use, sharing, adaptation, distribution and reproduction in any medium or format, as long as you give appropriate credit to the original author(s) and the source, provide a link to the Creative Commons licence, and indicate if changes were made. The images or other third party material in this article are included in the article's Creative Commons licence, unless indicated otherwise in a credit line to the material. If material is not included in the article's Creative Commons licence and your intended use is not permitted by statutory regulation or exceeds the permitted use, you will need to obtain permission directly from the copyright holder. To view a copy of this licence, visit http://creativecommons.org/licenses/by/4.0/.

\section{References}

1. Cancer Statistics for the UK (2019) Cancer Research UK. https:// www.cancerresearchuk.org/cancer-info/cancerstats. Accessed 1 June 2020

2. The Association of Coloproctology of Great Britain and Ireland (2007) Guidelines for the management of Colorectal Cancer. 3rd Edition. https://www.acpgbi.org.uk/content/uploads/2007-CCManagement-Guidelines.pdf. Accessed 1 June 2020.

3. Swellengrebel HA, Marijnen CA, Verwall VJ, Vincet A, Heuff G, Gerhards MF et al (2011) Toxicity and complications of preoperative chemoradiotherapy for locally advanced rectal cancer. $\mathrm{Br} \mathbf{J}$ Surg 98:418-426

4. West MA, Loughney L, Barben CP, Sripadam R, Kemp GJ, Grocott MP et al (2014) The effects of neoadjuvant chemoradiotherapy on physical fitness and morbidity in rectal cancer surgery patients. Eur J Surg Oncol 40:1421-1428

5. Joglekar S, Nau PN, Mezhir JJ (2015) The impact of sarcopenia on survival and complications in surgical oncology: a review of the current literature. J Surg Oncol 112:503-509

6. Jones KI, Doleman B, Scott S, Lund JN, Williams JP (2015) Simple psoas cross-sectional area measurement is a quick and easy method to assess sarcopenia and predicts major surgical complications. Colorectal Dis 17:O20-26

7. Miyamoto Y, Baba Y, Sakamoto Y, Ohuchi M, Tokunaga R, Kurashige J et al (2015) Sarcopenia is a negative prognostic factor after curative resection of colorectal cancer. Ann Surg Oncol 22:2663-2668 
8. Dolan DR, Knight KA, Maguire S, Moug SJ (2019) The relationship between sarcopenia and survival at 1 year in patients undergoing elective colorectal cancer surgery. Tech Coloproctol 23(9):877-885

9. Rinninella E, Cintoni M, Raoul P, Pozzo C, Strippoli A, Bria E et al (2019) Muscle mass, assessed at diagnosis by L3-CT scan as a prognostic marker of clinical outcomes in patients with gastric cancer: a systematic review and meta-analysis. Clin Nutr 19:33108-33115

10. Zhuang CL, Shen X, Zou HB, Dong QT, Cai HY, Chen XL et al (2019) EWGSOP2 versus EWGSOP1 for sarcopenia to predict prognosis in patients with gastric cancer after radical gastrectomy: analysis from a large-scale prospective study. Clin Nutr S0261-5614:33111-33115

11. Noguchi G, Kawahara T, Kobayashi K, Tsutsumi S, Ohtake S, Osaka K, Umemoto S, Nakaigawa N, Uemura H, Kishida T, Yao M (2020) A lower psoas muscle volume was associated with a higher rate of recurrence in male clear cell renal cell carcinoma. PLoS ONE 15:e0226581

12. Icard P, Schussler O, Loi M, Bobbio A, Lupo AM, Wislez M et al (2020) Pre-disease and pre-surgery BMI, weight loss and sarcopenia impact survival of resected lung cancer independently of tumor stage. Cancers 12:E266. https://doi.org/10.3390/cancers12020266

13. Lai CW, Minto G, Challand CP, Hosie KB, Sneyd JR, Creanor S et al (2013) Patients' inability to perform a preoperative cardiopulmonary exercise test or demonstrate an anaerobic threshold is associated with inferior outcomes after major colorectal surgery. Br J Anaesth 111:607-611

14. Carlisle J, Swart M (2007) Mid-term survival after abdominal aortic aneurysm surgery predicted by cardiopulmonary exercise testing. Br J Surg 94:966-969

15. West MA, Lythgoe D, Barben CP, Noble L, Kemp GJ, Jack S et al (2014) Cardiopulmonary exercise variables are associated with postoperative morbidity after major colonic surgery: a prospective blinded observational study. Br J Anaesth 112:665-671

16. West MA, Parry MG, Lythgoe D, Barben CP, Kemp GJ, Grocott MP et al (2014) Cardiopulmonary exercise testing for the prediction of morbidity risk after rectal cancer surgery. Br J Surg 101:1166-1172

17. Moran J, Wilson F, Guinan E, McCormick P, Hussey J, Moriarty J (2016) Role of cardiopulmonary exercise testing as a risk-assessment method in patients undergoing intra-abdominal surgery: a systematic review. Br J Anaesth 116:177-191

18. Carli F, Charlebois P, Stein B, Feldman L, Zavorsky G, Kim DJ et al (2010) Randomized clinical trial of prehabilitation in colorectal surgery. Br J Surg 97:1187-1197

19. West MA, Loughney L, Lythgoe D, Barben CP, Sripadam R, Kemp GJ et al (2015) Effect of prehabilitation on objectively measured physical fitness after neoadjuvant treatment in preoperative rectal cancer patients: a blinded interventional pilot study. Br J Anaesth 114:244-251

20. Mayo NE, Feldman L, Scott S, Zavorsky G, do Kim J, Charlebois $P$ et al (2011) Impact of perioperative change in physical function on postoperative recovery: argument supporting prehabilitation for colorectal surgery. Surgery 150:505-514

21. Moran J, Guinan E, McCormick P, Larkin J, Mockler D, Hussey J et al (2016) The ability of prehabiliation to influence postoperative outcome after intra-abdominal operation: a systematic review and meta-analysis. Surgery 160:1189-1201

22. Barberan-Garcia A, Ubre M, Roca J, Lacy AM, Burgos F, Risco R et al (2018) Personalised prehabilitation in high-risk patients undergoing elective major abdominal surgery. Ann Surg 267:50-56

23. Moug SJ, Mutrie N, Barry SJE, Mackay G, Steele RJC, Boachie $\mathrm{C}$ et al (2019) Prehabilitation is feasible in patients with rectal cancer undergoing neoadjuvant chemoradiotherapy and may minimize physical deterioration: results from the REx trial. Colorectal Dis 21:548-562

24. Scottish Executive Let's make Scotland more active (2003) Edinburgh: Scottish Executive. https://www.paha.org.uk/Resource/ lets-make-scotland-more-active. Accessed 1 June 2020.

25. Tudor-Locke C, Bassett DR Jr (2004) How many steps/ day are enough? Preliminary pedometer induces for public health. Sports Med 34:1-8

26. Baker G, Gray SR, Wright A, Fitzsimons C, Nimmo M, Lowry $R$ et al (2008) The effect of a pedometer-based community walking intervention "Walking for Wellbeing in the West" on physical activity levels and health outcomes: a 12-week randomized controlled trial. Int J Behav Nutr Phys Act 5:44

27. Croteau KA, Richeson NE, Farmer BC, Jones DB (2007) Effect of a pedometer-based intervention on daily step counts of community-dwelling older adults. Res Q Exerc Sport 78:401-406

28. Fitzsimons C, Baker G, Gray S, Nimmo M, Mutrie N, The Scottish Physical Activity Research Collaboration (2012) Does physical activity counselling enhance the effects of a pedometer-based intervention over the long-term: 12-month findings from the Walking for Wellbeing in the West study. BMC Public Health 12:206

29. Prado C, Lieffers J, McCargar L, Reiman T, Sawyer M, Martin L et al (2008) Prevalence and clinical implications of sarcopenic obesity in patients with solid tumours of the respiratory and gastrointestinal tracts: a population-based study. Lancet Oncol 9:629-635

30. R Core Team (2018) R: a language and environment for statistical computing. R Foundation for Statistical Computing, Vienna, Austria. https://www.R-project.org/.

31. Malietzis G, Currie AC, Athanasiou T et al (2016) Influence of body composition profile on outcomes following colorectal cancer surgery. Br J Surg 103:572-580

32. Pring ET, Malietzis G, Kennedy RH, Athanasiou T, Jenkins JT (2018) Cancer cachexia and myopenia: update on management strategies and the direction of future research for optimising body composition in cancer. A narrative review. Cancer Treat Review 70:245-254

33. World Cancer Research Fund International (2019) Continuous Update Project https://www.wcrf.org/int/continuous-update-proje ct. Accessed 1 June 2020.

34. Cruz-Jentoft AJ, Bahat G, Bauer J et al (2019) Sarcopenia: revised European consensus on definition and diagnosis. Age Ageing 48:16-31

35. Liao C-D, Chen H-C, Huang S-W, Liou T-H (2019) The role of muscle mass gain following protein supplementation plus exercise therapy in older adults with sarcopenia and frailty risks: a systematic review and meta-regression analysis of randomized trials. Nutrients 11:1713-1726

36. Beckwee D, Delaere A, Albrecht S et al (2019) Exercise interventions for the prevention and treatment of sarcopenia. A systematic umbrella review. J Nutr Health Aging 23:494-502

37. Malietzis G, Currie AC, Johns N et al (2016) Skeletal muscle changes after elective colorectal cancer resection: a longitudinal study. Ann Surg Oncol 23:2539-2547

38. Jones LW (2015) Precision oncology framework for investigation of exercise as treatment for cancer. J Clin Oncol 33:4134-4137

39. Ticinesi A, Nouvenne A, Cerundolo N et al (2019) Gut microbiota, muscle mass and function in aging: a focus on physical frailty and sarcopenia. Nutrients 11:1633-1654

40. Prehabilitation Evidence and Insight Review. Macmillan Cancer Support (2019) https://www.macmillan.org.uk/_images/prehabilit ation-evidence-and-insight-review_tcm9-335025.pdf. Accessed July 2019

Publisher's Note Springer Nature remains neutral with regard to jurisdictional claims in published maps and institutional affiliations. 